\title{
THE ASYMPTOTIC-NORMING AND THE RADON-NIKODYM PROPERTIES ARE EQUIVALENT IN SEPARABLE BANACH SPACES
}

\author{
N. GHOUSSOUB AND B. MAUREY
}

\begin{abstract}
We show that the asymptotic-norming and the Radon-Nikodym properties are equivalent, settling a problem of James and Ho [9]. In the process, we give a positive solution to two questions of Edgar and Wheeler [6] concerning Cech-complete Banach spaces. We also show that a separable Banach space with the Radon-Nikodym property semi-embeds in a separable dual whenever it has a norming space not containing an isomorphic copy of $l_{1}$. This gives a partial answer to a problem of Bourgain and Rosenthal [3].
\end{abstract}

Introduction. Let $X$ be a Banach space. We recall that $X$ is said to have the point of continuity property (resp. the Radon-Nikodym property) if every weakly closed bounded subset of $X$ has a point of weak to norm continuity (resp. a denting point). A separable Banach space $X$ is said to have the asymptotic norming property if there exists a separable Banach space $Y$ such that $X$ is (isomorphic to) a subspace of $Y^{*}$ which verifies the following property:

$$
\begin{aligned}
& \text { if }\left(x_{n}\right) \subseteq X, x_{n} \stackrel{w^{*}}{\rightarrow} y^{*} \text { and }\left\|x_{n}\right\| \rightarrow\left\|y^{*}\right\|, \\
& \text { then } \lim _{n}\left\|x_{n}-y^{*}\right\|=0 .
\end{aligned}
$$

In [9], James and Ho introduced the asymptotic norming property, proved that it implies the Radon-Nikodym property and asked whether the two properties are equivalent. To prove this conjecture we recall that Davis and Johnson [5] showed that for every separable subspace $X$ of $Y^{*}$, the latter can be renormed in such a way that the conclusion of (A.N.P.) holds provided $y^{*}$ is assumed to be in $X$. The only missing ingredient in the equivalent norm is then the term that forces $y^{*}$ to be in $X$. On the other hand, the authors proved in [7] the following

THEOREM 1 [7]. Let $X$ be a separable Banach space. Then $X$ has the point of continuity property (resp. the Radon-Nikodym property) if and only if $X$ embeds isometrically in the dual of a separable Banach subspace $Y$ of $X^{*}$ in such a way that $Y^{*} \backslash X=\bigcup_{n} K_{n}$ where each $K_{n}$ is weak*-compact (resp. weak*-compact and convex) in $Y^{*}$.

It is easy to see that if the convex $K_{n}$ 's can be chosen to be a strictly positive distance away from $X$, then the distance of the elements of $Y^{*}$ to the $K_{n}$ 's (made into a suitable seminorm) would give the missing ingredient that forces $y^{*}$ to be in $X$.

Received by the editors July 16, 1984.

1980 Mathematics Subject Classification. Primary 46G10, 46B22.

Key words and phrases. Asymptotic-norming and Radon-Nikodym properties, semi-embeddings. 
The problem of the existence of such $K_{n}$ 's coincide with a question of Edgar and Wheeler [6] on strongly Cech-complete Banach spaces. The following theorem gives a positive answer to these questions.

THEOREM (1) BIS. Let $X$ be a separable Banach space. Then $X$ has the point of continuity property (resp. the Radon-Nikodym property) if and only if $X$ embeds in the dual of a separable Banach space $Y$ in such a way that $Y^{*} \backslash X=\bigcup_{n} K_{n}$ where each $K_{n}$ is weak*-compact (resp. weak*-compact and convex) satisfying $d\left(K_{n}, X\right)>$ 0 .

The proof will be broken into several lemmas. We shall need the following notations and terminology: If $C$ is a subset of a dual space $Y^{*}$ we shall denote by $\bar{C}^{*}$ (resp. $\bar{C}$ ) its weak*-closure (resp. its norm closure). The distance between two subsets $C$ and $D$ of $Y^{*}$ will be denoted by $d(C, D)=\inf \{\|x-y\| ; x \in C, y \in D\}$. If $\Delta$ is any metric on $Y^{*}, l \in Y^{*}$ and $\rho>0$, then $B_{\Delta}(l, \rho)$ will be the $\Delta$-open ball $\{y \in Y ; \Delta(y, l)<\rho\}$. If $\Delta$ is induced by the norm we shall simply write $B(l, \rho)$. The closed unit ball of a Banach space $Z$ will be denoted $B_{Z}$.

If now $X$ is a subspace of $Y^{*}$ and $L$ is a $w^{*}$-compact subset of $Y^{*}$ which is disjoint from $X$, we shall say that $L$ is $\rho$-bad with respect to $X$ for some $\rho>0$ if

(i) for each $\varepsilon>0$, the set $L_{\varepsilon}=\{l \in L ; d(l, X) \leq \varepsilon\}$ is $w^{*}$-dense in $L$;

(ii) the set $L^{\rho}=\{l \in L ; d(l, X) \geq \rho\}$ is also $w^{*}$-dense in $L$.

LEMMA (1). Let $X$ be a subspace of the dual of a separable Banach space $Y$, such that $\bar{B}_{X}^{*}=B_{Y^{*}}$. Let $L$ be a $w^{*}$-compact subset of $\theta \cdot B_{Y^{*}}(\theta<1)$ which is $\rho$-bad with respect to $X$ for some $\rho>0$. Let $K$ be a $w^{*}$-compact subset of $B_{Y^{*}}$ which is disjoint from $X$. Then for each $\varepsilon$ such that $0<\varepsilon<1-\theta$ and each $w^{*}$-open subset $V$ of $B_{Y^{*}}$ with $V \cap L \neq \emptyset$, there exist $l \in L, x \in X$ such that

(i) $\|x\| \leq \varepsilon$,

(ii) $l+x \in V$,

(iii) $l+x \notin K$,

(vi) $d(l+x, X) \geq \rho-\varepsilon$.

Proof. Choose $l_{1}$ in $L$ such that $l_{1} \in V$ and $d\left(l_{1}, X\right)<\varepsilon / 2$. Consider $x_{1}$ and then $\left(y_{n}\right)$ in $X$ such that $l_{1}=w^{*}-\lim _{n}\left(x_{1}+y_{n}\right)$ with $\left\|y_{n}\right\| \leq \varepsilon / 2$. Note that $\left\|x_{1}\right\| \leq \theta+\varepsilon / 2$, hence $x_{1}+y_{n} \in B_{X}$. Choose now $n_{0}$ such that $x_{1}+y_{n_{0}} \in V$. We have that $x_{1}+y_{n_{0}} \notin K$. On the other hand, $w^{*}-\lim _{n}\left(l_{1}-y_{n}+y_{n_{0}}\right)=x_{1}+y_{n_{0}}$ and $\left\|l_{1}-y_{n}+y_{n_{0}}\right\| \leq 1$, hence, for a large enough $n_{1}$, we have $\left(l_{1}-y_{n_{1}}+y_{n_{0}}\right) \in V$ and $\left(l_{1}-y_{n_{1}}+y_{n_{0}}\right) \notin K$.

Since $L$ is $\rho$-bad, choose $\left(l_{m}\right) \subseteq L, d\left(l_{m}, X\right) \geq \rho$ such that $l_{1}=w^{*}-\lim \left(l_{m}\right)$. That is, for a large enough $m_{0}$, we have $\left(l_{m_{0}}-y_{n_{1}}+y_{n_{0}}\right) \in V$ and $\left(l_{m_{0}}-y_{n_{1}}+y_{n_{0}}\right) \notin K$. Now take $l=l_{m_{0}}$ and $x=\left(-y_{n_{1}}+y_{n_{0}}\right)$. They clearly verify the claimed properties.

LEMMA (2). Let $X$ be a separable subspace of the dual of a separable Banach space $Y$ such that $B_{X}$ is a $w^{*}-G_{\delta}, w^{*}$-dense in $B_{Y^{*}}$. Then for each $\theta<1, \theta B_{Y^{*}}$ contains no $\rho$-bad $w^{*}$-compact sets with respect to $X$ for any $\rho>0$.

PROOF. Write $B_{Y^{*}} \backslash B_{X}=\bigcup_{n} K_{n}$ where $\left(K_{n}\right)$ is an increasing sequence of $w^{*}$ compact sets. Let $\Delta$ be a distance defining the $w^{*}$-topology on $B_{Y^{*}}$ and let $\left(z_{n}\right)$ be a dense sequence in $B_{X}$. Suppose $L$ is a weak* ${ }^{*}$-compact subset of $\theta \cdot B_{Y^{*}}(\theta<1)$ which is $\rho$-bad with respect to $X$ for some $\rho>0$. 
Let $l_{0}$ be any point in $L$ and let $V_{0}=B_{\Delta}\left(l_{0}, 1\right)$ and $0<\varepsilon_{0}<\inf (1-\theta, \rho / 2)$. Use Lemma (1) to obtain $x_{0} \in X,\left\|x_{0}\right\| \leq \varepsilon_{0}$ and $l_{0}^{\prime} \in L$ such that $l_{0}^{\prime}+x_{0} \in V_{0}$, $l_{0}^{\prime}+x_{0} \notin K_{0}$ and $d\left(l_{0}^{\prime}+x_{0}, X\right) \geq \rho-\varepsilon_{0}>\rho / 2$.

Now set $L_{1}=L+x_{0}, \theta_{1}=\theta+\varepsilon_{0}<1$ and $l_{1}=l_{0}^{\prime}+x_{0}$.

Note that $L_{1}$ is also $\rho$-bad with respect to $X$ and $L_{1} \subseteq \theta_{1} \cdot B_{Y^{*}}$. Let $V_{1}^{\prime}$ be a $w^{*}$-open subset of $V_{0}$ containing $l_{1}$ such that $\bar{V}_{1}^{\prime} \cap\left(K_{0} \cup \bar{B}^{*}\left(z_{0}, \rho / 2\right)\right)=\emptyset$. Set $V_{1}=V_{1}^{\prime} \cap B_{\Delta}\left(l_{1}, 1 / 2\right)$ and $\varepsilon_{1}<\inf \left(1-\theta_{1}, \rho / 2\right)$ and apply again Lemma 1 to obtain $x_{1} \in X,\left\|x_{1}\right\| \leq \varepsilon_{1}, l_{1}^{\prime} \in L_{1}, l_{1}^{\prime}+x_{1} \in V_{1}, l_{1}^{\prime}+x_{1} \notin K_{1}$ and $d\left(l_{1}^{\prime}+x_{1}, X\right) \geq \rho-\varepsilon_{1}>$ $\rho / 2$.

By induction, we get a decreasing sequence $\left(V_{n}\right)$ of $w^{*}$-open subsets of $B_{Y^{*}}$ and a sequence $\left(l_{n}\right)$ of vectors such that

(i) $l_{n} \in V_{n}$ for each $n$,

(ii) $\operatorname{diam}_{\Delta}\left(\bar{V}_{n}^{*}\right) \leq 2^{-n}$,

(iii) $\bar{V}_{n}^{*} \cap\left(K_{n-1} \cup\left(\bigcup_{j=0}^{n-1} \bar{B}^{*}\left(z_{j}, \rho / 2\right)\right)\right)=\emptyset$.

It follows that the $w^{*}$-limit $l_{\infty}$ of $\left(l_{n}\right)$ can neither be in $B_{X}$ nor in any of the $K_{n}$ 's, which is obviously a contradiction since $l_{\infty} \in B_{Y^{*}}$.

LEMMA (3). Let $X$ be a separable subspace of the dual of a separable Banach space $Y$ such that $B_{X}$ is a $w^{*}-G_{\delta}, w^{*}$-dense in $B_{Y^{*}}$. Let $L$ be a subset of $Y^{*}$ which is disjoint of $X$. Then:

(i) If $L$ is $w^{*}$-compact, there exists a $w^{*}$-open set $V$ such that $L \cap V \neq \emptyset$ and $d\left(L \cap \bar{V}^{*}, X\right)>0$.

(ii) If $L$ is $w^{*}$-compact and convex, there exists a $w^{*}$-open half-space $V$ such that $L \cap V \neq \emptyset$ and $d\left(L \cap \bar{V}^{*}, X\right)>0$.

ProOF. We first claim that there exists $\varepsilon>0$ such that the set $L_{\varepsilon}=\{l \in L$; $d(l, X) \leq \varepsilon\}$ is not $w^{*}$-dense in $L$. Indeed, suppose not. We can assume without loss that $L \subseteq B_{Y^{*}} / 2$. Now note that $L=\bigcup_{n} L^{n}$ where $L^{n}=\{l ; d(l, X) \geq 1 / n\}$ since $L \cap X=\emptyset$. It follows that there exists $m$ such that ${\overline{L^{m}}}^{*}$ has a nonempty interior $V_{0}$ in the $w^{*}$-topology relative to $L$. It follows that $\bar{V}_{0}^{*}$ is a $1 / m$-bad set with respect to $X$ which clearly contradicts Lemma (2).

In case (i) we take $V$ to be a $w^{*}$-open subset of $Y^{*}$ such that $V \cap L=L \backslash \bar{L}_{\varepsilon}^{*}$ which is nonempty.

In case (ii), note that $\bar{L}_{\varepsilon}^{*}$ is also convex, hence, any $w^{*}$-open half-space $V$ that separates any point $l$ in $L \backslash \bar{L}_{\varepsilon}^{*}$ from $\bar{L}_{\varepsilon}^{*}$ will do the job.

LEMMA (4). Let $X$ be a separable subspace of the dual of a separable Banach space $Y$ such that $B_{X}$ is a $w^{*}-G_{\delta}$ set which is $w^{*}$-dense in $B_{Y^{*}}$. Let $L$ be a subset of $Y^{*}$ which is disjoint of $X$. Then:

(i) If $L$ is $w^{*}$-compact, there exists a countable collection of $w^{*}$-compact sets $\left(L_{n}\right)$ whose union is $L$ such that $d\left(L_{n}, X\right)>0$ for each $n$.

(ii) If $L$ is $w^{*}$-compact and convex, there exists a countable collection of $w^{*}$ compact convex sets whose union is $L$ such that $d\left(L_{n}, X\right)>0$ for each $n$.

ProOF. (i) By transfinite induction, we define a decreasing family $\left(K_{\alpha}\right)$ of $w^{*}$-compact subsets of $L$ in the following manner:

(a) $K_{0}=L$.

(b) If $\alpha=\beta+1$ and $K_{\beta}$ nonempty, apply Lemma (3) to $K_{\beta}$ to obtain a $w^{*}$-open set $V_{\beta}$ such that $K_{\beta} \cap V_{\beta} \neq \emptyset$ and $d\left(K_{\beta} \cap \bar{V}_{\beta}^{*}, X\right)>0$. Set $K_{\alpha}=K_{\beta} \backslash V_{\beta}$. 
(c) If $\alpha$ is a limit ordinal, set $K_{\alpha}=\bigcap_{\beta<\alpha} K_{\beta}$.

Since $L$ is $w^{*}$-metrizable there exists $\gamma<\Omega$ (the first uncountable ordinal) such that $K_{\gamma}=\emptyset$. It is clear that $L=\bigcup_{\alpha<\gamma} K_{\alpha} \cap \bar{V}_{\alpha}^{*}$ and $L_{\alpha}=K_{\alpha} \cap \bar{V}_{\alpha}^{*}$ is a strictly positive distance away from $X$ for each $\alpha<\gamma$.

(ii) If $L$ is also convex, then $V$ can be taken to be a $w^{*}$-open half-space by Lemma (3), hence each $L_{\alpha}=K_{\alpha} \cap \bar{V}_{\alpha}^{*}$ is then $w^{*}$-compact and convex.

The following is now immediate:

THEOREM (1) TER. Let $X$ be a separable subspace of the dual of a separable Banach space $Y$ such that $B_{X}$ is $w^{*}$-dense in $B_{Y^{*}}$. If $Y^{*} \backslash X=\bigcup_{n} K_{n}$ where each $K_{n}$ is $w^{*}$-compact (resp. $w^{*}$-compact and convex), then $Y^{*} \backslash X=\bigcup_{n} K_{n}^{\prime}$ where each $K_{n}^{\prime}$ is $w^{*}$-compact (resp. $w^{*}$-compact and convex) such that $d\left(K_{n}^{\prime}, X\right)>0$.

Proof OF THEOREM (1) BIS. If $X$ is a separable Banach space with the point of continuity property, apply Theorem (1) to get a separable Banach subspace $Y$ of $X^{*}$ such that $X$ is a subspace of $Y^{*}$ verifying $Y^{*} \backslash X=\bigcup_{n} K_{n}$ where each $K_{n}$ is $w^{*}$-compact. It follows that $B_{X}$ is a $w^{*}-G_{\delta}, w^{*}$-dense subset of $B_{Y^{*}}$. Apply now Theorem (1) ter to get the conclusion.

If $X$ has the Radon-Nikodym property, each $K_{n}$ is then convex, and Theorem (1) ter applies again and gives the claimed result.

The following corollary answers two questions of Edgar and Wheeler [6]:

COROllary (5). (a) A separable Banach space $X$ has the point of continuity property and its dual $X^{*}$ is separable if and only if $X^{* *} \backslash X$ is the countable union of $w^{*}$-compact sets $\left(K_{n}\right)$ such that $d\left(K_{n}, X\right)>0$.

(b) A separable Banach space $X$ has the Radon-Nikodym property and its dual $X^{*}$ is separable if and only if $X^{* *} \backslash X$ is the countable union of $w^{*}$-compact convex sets $\left(K_{n}\right)$ such that $d\left(K_{n}, X\right)>0$.

PROOF. In view of the results of [6 and 7] the space $Y$ mentioned in Theorem (1) can be taken in this case to be the separable dual $X^{*}$.

Theorem (1) bis and the proof of Theorem 4.14 of [6] applied to $Y$ instead of $X^{*}$ gives the following

COROLlaRY (6). A separable Banach space $X$ has the point of continuity property if and only if there exists a separable Banach space $Y$ and a family of norm one vectors $\left\{y_{n, i} ; 1 \leq i \leq m_{n}, n \in \mathbf{N}\right\}$ in $Y$ such that

$$
X=\left\{y^{*} \in Y^{*} ; \frac{\lim }{n} \max _{1 \leq i \leq m_{n}}\left|y^{*}\left(y_{n, i}\right)\right|=0\right\} \text {. }
$$

The following settles a question of James and Ho [9]:

THEOREM (2). A separable Banach space $X$ has the Radon-Nikodym property if and only if it has the asymptotic-norming property.

Proof. Suppose that $X$ has the Radon-Nikodym property. Apply Theorem (1) bis to obtain a separable Banach space $Y$ such that $X$ is a subspace of $Y^{*}$ verifying $Y^{*} \backslash X=\bigcup_{n} K_{n}$ where each $K_{n}$ is $w^{*}$-compact convex and $d\left(K_{n}, X\right) \geq$ $\varepsilon_{n}>0$. Following Davis and Johnson [5], let $\left(E_{n}\right)_{n}$ be an increasing sequence of 
finite-dimensional subspaces of $X$ such that $X=\overline{\bigcup_{n} E_{n}}$ and define the seminorm $\||| x \mid\|=\sum_{n} 2^{-n} d\left(x, E_{n}\right)$. Now let ! ! $n$ be the seminorm defined by

$$
! x !_{n}=d\left(x, \mathbf{R}_{+} K_{n}\right)+d\left(x,-\mathbf{R}_{+} K_{n}\right)
$$

and set $! x !=\sum_{n} 2^{-n} ! x !_{n}$. Finally, let $\|x\|_{1}=\|x\|+\|x \mid\|+! x !$. Note that $\|x\| \leq$ $\|x\|_{1} \leq 7\|x\|$ for each $x$ in $Y^{*}$ and that \|\|$_{1}$ is $w^{*}$-lower semicontinuous, hence, it is a dual norm on $Y^{*}$.

Suppose now that $\left(x_{n}\right) \subseteq X, y^{*} \in Y^{*}$ such that $\left\|x_{n}\right\|_{1} \rightarrow\left\|y^{*}\right\|_{1}$ and $w^{*}$ $\lim _{n}\left(x_{n}\right)=y^{*}$. Since each piece of the norm is $w^{*}$-lower semicontinuous we get that $\left\|x_{n}\right\| \rightarrow\left\|y^{*}\right\|,\left\||| x_{n}\right\|\|\rightarrow\| y^{*} \| \mid$ and $d\left(x_{n}, \mathbf{R}_{+} K_{m}\right) \rightarrow d\left(y^{*}, \mathbf{R}_{+} K_{m}\right)$ for each $m$.

We claim that $y^{*} \in X$. Indeed, if not, then there exists an $m$ such that $y^{*} \in K_{m}$ and $\lim _{n} d\left(x_{n}, \mathbf{R}_{+} K_{m}\right)=d\left(y^{*}, \mathbf{R}_{+} K_{m}\right)=0$.

We can then suppose that $\left\|x_{n}-\lambda_{n} k_{n}\right\| \leq 1 / n$ for some $\lambda_{n} \in \mathbf{R}_{+}$and $k_{n} \in K_{m}$. This gives

$$
1 / n \geq\left\|x_{n}-\lambda_{n} k_{n}\right\|=\lambda_{n}\left\|x_{n} / \lambda_{n}-k_{n}\right\| \geq \lambda_{n} \varepsilon_{m} .
$$

It follows that $\lambda_{n} \rightarrow 0$ and $\left\|x_{n}\right\| \rightarrow 0$, a contradiction. Since $y^{*}$ is now in $X$, the Davis-Johnson norm insures that $\lim _{n}\left\|x_{n}-y^{*}\right\|=0$.

The converse was proved by James and Ho [9]. We sketch an easier proof based on martingales and already used by Davis et al. [4]. Let $D$ be a countable dense set in the unit ball of $Y$. Let $\left(\phi_{n}\right)$ be an $X$-valued bounded martingale. Let $\phi_{\infty}$ be a $w^{*}$-limit of $\left(\phi_{n}\right)$ which is valued in $Y^{*}$. For each $y \in D$, the real-valued martingale $y\left(\phi_{n}\right)$ converges to $y\left(\phi_{\infty}\right)$ outside a set $\Omega_{y}$ of measure zero.

By a lemma of Neveu [11], the martingale $\left\|\phi_{n}\right\|=\sup _{y \in D}\left|y\left(\phi_{n}\right)\right|$ converges to $\sup _{y \in D}\left|y\left(\phi_{\infty}\right)\right|=\left\|\phi_{\infty}\right\|$ outside a set $\Omega_{0}$ of measure zero. Since $X$ has the asymptotic norming property with respect to $Y$, we get that $\lim _{n \rightarrow \infty}\left\|\phi_{n}-\phi_{\infty}\right\|=0$ outside the set $\Omega_{0} \cup \bigcup_{y \in D} \Omega_{y}$ which is of measure zero.

Recall that a bounded linear operator $T$ from a Banach space $X$ into a space $Y$ is said to be a semi-embedding if it is one-to-one and if the image of the unit ball of $X$ by $T$ is norm closed in $Y$. In [3], Bourgain and Rosenthal showed that the $\mathcal{L}_{\infty}$-spaces constructed by Bourgain and Delbaen [2] do not semi-embed in separable duals even though they enjoy the Radon-Nikodym property. On the other hand, they show that the Radon-Nikodym spaces constructed by Johnson and Lindenstrauss [10] do semi-embed in separable duals even though they do not embed in such spaces. The following theorem gives a sufficient condition that guarantees such semi-embeddings for Radon-Nikodym spaces. It gives a partial solution to a question of Bourgain and Rosenthal [3].

THEOREM (3). If $X$ is a separable Banach space with a norming space not containing an isomorphic copy of $l_{1}$, then $X$ has the Radon-Nikodym property if and only if it semi-embeds in a separable dual.

First we need the following

LEMMA (5). Let $Y$ be a separable Banach space not containing an isomorphic copy of $l_{1}$. If $X$ is a separable subspace of $Y^{*}$ with the Radon-Nikodym property such that $B_{X}$ is $w^{*}$-dense in $B_{Y^{*}}$, then the orthogonal of $X$ in $Y^{* *}$ is $w^{*}$-separable.

PROOF. By a theorem of Bourgain [1], $B_{X}$ is then $w^{*}$-dentable in $Y^{*}$; that is, every norm closed convex subset of $B_{X}$ contains $w^{*}$-open slices with arbitrarily 
small diameters. Now, we proceed as in Lemma III.1 of [7]: Fix $\varepsilon>0$ and define inductively a decreasing family of norm-closed convex subsets $\left(F_{\alpha}\right)$ of $B_{X}$ in the following way:

(i) $F_{0}=B_{X}$.

(ii) If $\alpha=\beta+1$ and $F_{\beta} \neq \emptyset$, use the $w^{*}$-dentability to find a $w^{*}$-open slice $S_{\beta}$ of $F_{\beta}$ such that $\operatorname{diam}\left(S_{\beta}\right)<\varepsilon$. Set $F_{\alpha}=F_{\beta} \backslash S_{\beta}$.

(iii) If $\alpha$ is a limit ordinal, let $F_{\alpha}=\bigcap_{\beta<\alpha} F_{\beta}$.

Since $B_{X}$ is separable, there exists $\gamma<\Omega$ (the first uncountable ordinal) such that $F_{\gamma}=\emptyset$ and $F_{\beta} \neq \emptyset$ for $\beta<\gamma$. Let $K_{\alpha}$ be the $w^{*}$-closure of $F_{\alpha}$ in $Y^{*}$ and let $H_{\alpha}$ be the $w^{*}$-open half-space such that $S_{\alpha}=H_{\alpha} \cap F_{\alpha}$. It is clear that

$$
B_{X} \subseteq \bigcap_{\alpha \leq \gamma}\left(K_{\alpha} \cup \bigcup_{\beta<\alpha} H_{\beta}\right) .
$$

Moreover, if $x$ belongs to the set on the right-hand side, then $x \in K_{\beta} \cap H_{\beta}$ for some $\beta<\gamma$ which implies that $d\left(x, B_{X}\right) \leq \varepsilon$. It follows that if we repeat the construction for each $\varepsilon=1 / n$ we then get

$$
B_{X}=\bigcap_{n} \bigcap_{\alpha \leq \gamma_{n}}\left(K_{\alpha, n} \cup \bigcup_{\beta<\alpha} H_{\beta, n}\right) \text {. }
$$

Since $Y$ is separable, write that $K_{\alpha, n}=\bigcap_{m} L_{\alpha, n, m}$ where each $L_{\alpha, n, m}$ is a $w^{*}$ open half-space in $Y^{*}$. It follows that $Y^{*} \backslash B_{X}$ and hence $Y^{*} \backslash X$ is a countable union of $w^{*}$-compact convex subsets $\left(K_{n}\right)$ of $Y^{*}$. By Theorem (1) ter, we can suppose that $d\left(K_{n}, X\right)>\varepsilon_{n}>0$. If $\pi$ is now the quotient map from $Y^{*}$ onto $Y^{*} / X$, we obtain that $0 \notin \overline{\pi\left(K_{n}\right)}$ for each $n$, hence, there exists $f_{n}$ in $\left(Y^{*} / X\right)^{*}=X^{\perp}$ such that $f_{n}>\varepsilon_{n}$ on $\overline{\pi\left(K_{n}\right)}$. It is now clear that $X=\left\{y^{*} \in Y^{*} ; f_{n}\left(y^{*}\right)=0 ; \forall n \in \mathbf{N}\right\}$ and that $X^{\perp}$ is $w^{*}$-separable.

PROOF OF THEOREM (3). Since $l_{1}$ does not embed in $Y$, use Odell and Rosenthal's theorem [12] to find for each $n$ a sequence $\left(g_{n, m}\right)$ in $Y$ that converges pointwise on $Y^{*}$ to $f_{n}$. The space $X$ can now be written as $\left\{y^{*} \in Y^{*}\right.$; $\left.\lim _{m \rightarrow \infty} g_{n, m}\left(y^{*}\right)=0 ; \forall n \in \mathbf{N}\right\}$. We may suppose that $\left\|g_{n, m}\right\| \leq 1$ for each $n, m \in$ N. Define now for each $n \geq 1$, the operator $T_{n}: l_{1} \rightarrow Y$ by $T_{n}\left(\alpha_{m}\right)_{m}=\sum_{m} \alpha_{m} g_{n, m}$ and let $T_{0}: l_{2} \rightarrow Y$ be a dense range operator. Let $T: l_{2} \oplus\left(\sum_{n} \oplus l_{1}\right)_{l_{2}} \rightarrow Y$ be the unique linear operator whose restrictions to the factor spaces are those given by the sequence $\left\{T_{0},\left(2^{-n} T_{n}\right)_{n \geq 1}\right\}$. Let $T^{*}: Y^{*} \rightarrow l_{2} \oplus\left(\sum_{n} \oplus l_{\infty}\right)_{l_{2}}$ be the dual operator which is one-to-one since $T_{0}^{*}$ is. We claim that $T^{*}$ is valued in $l_{2} \oplus\left(\sum_{n} \bigoplus c\right)_{l_{2}}$ and that $T^{*}\left(B_{X}\right)$ is norm-closed. Indeed, we have for each $n \geq 1$ and each $y^{*} \in Y^{*}$, $\left(T_{n}^{*}\left(y^{*}\right)\right)=\left(g_{n, m}\left(y^{*}\right)\right)_{m}$ which is convergent to $f_{n}\left(y^{*}\right)$. Moreover, if $x_{l} \in B_{X}$ and $\lim _{l} T^{*} x_{l}=z$, then $z=T^{*} y^{*}$ where $y^{*}$ is a $w^{*}$-limit of $\left(x_{l}\right)$ in $Y^{*}$ since $T^{*}$ is oneto-one and $w^{*}$-to- $w^{*}$ continuous. Moreover, since for each $n, g_{n, m}\left(x_{l}\right) \rightarrow g_{n, m}\left(y^{*}\right)$ uniformly in $m$, we get that $f_{n}\left(x_{l}\right) \rightarrow f_{n}\left(y^{*}\right)$ and $f_{n}\left(y^{*}\right)=0$ for each $n$. It follows that $y^{*} \in B_{X}$.

The operator $T$ has a separable adjoint, hence the Stegall factorization theorem [13] applies and we get a separable Banach space $Z$ with a separable dual such that $T=U \circ V$ where $U: Z \rightarrow Y$ and $V: l_{2} \oplus\left(\sum_{n} \bigoplus l_{1}\right)_{l_{2}} \rightarrow Z$. Note now that $U^{*}\left(B_{X}\right)$ is norm-closed in $Z^{*}$ since $T^{*}\left(B_{X}\right)$ is closed in $l_{2} \oplus\left(\sum_{n} \bigoplus l_{\infty}\right) l_{2}$. Hence $U^{*}$ is a semi-embedding of $X$ into the separable dual $Z^{*}$. 
REMARK. The proof of Theorem (1) bis relies heavily on the fact that the ball of $X$ is a $w^{*}-G_{\delta}$ in $Y^{*}$. Actually the local statement is not true. In a forthcoming paper, we construct a $w^{*}-G_{\delta}$ subset $C$ of a dual space whose complement is not decomposable into a countable union of $w^{*}$-compact sets which are a strictly positive distance away from $C$. This question is closely related to the problem of minimizing a certain class of functions on the set $C$. We shall deal with these questions in [8].

\section{REFERENCES}

1. J. Bourgain, Sets with the Radon-Nikodym property in conjugate Banach spaces, Studia Math. 66 (1978), 199-205.

2. J. Bourgain and F. Delbaen, $A$ special class of $\mathcal{L}^{\infty}$-spaces, Acta Math. 145 (1980), 155-176.

3. J. Bourgain and H. P. Rosenthal, Applications of the theory of semi-embeddings to Banach space theory, J. Funct. Anal. 52 (1983), 149-188.

4. W. Davis, N. Ghoussoub and J. Lindenstrauss, A lattice renorming theorem and applications to vector-valued processes, Trans. Amer. Math. Soc. 263 (1981), 531-540.

5. W. Davis and W. B. Johnson, A renorming of non-reflexive Banach spaces, Proc. Amer. Math. Soc. 37 (1973), 486-487.

6. C. A. Edgar and R. F. Wheeler, Topological properties of Banach spaces, Pacific J. Math. 115 (1984), 317-350.

7. N. Ghoussoub and B. Maurey, $G_{\delta}$-embeddings in Hilbert space, J. Funct. Anal. (to appear).

8.,$H_{\delta}$-embeddings in Hilbert space and optimization on $G_{\delta}-$ sets, Mem. Amer. Math. Soc. (to appear).

9. R. C. James and A. Ho, The asymptotic-norming and Radon-Nikodym properties for Banach spaces, Ark. Mat. 19 (1981), 53-70.

10. W. B. Johnson and Y. Lindenstrauss, Examples of $\mathcal{L}^{1}$-spaces, Ark. Mat. 18 (1980), 101-106.

11. J. Neveu, Discrete parameter martingales, North-Holland, Amsterdam, 1975.

12. T. Odell and H. P. Rosenthal, A double dual characterization of separable Banach spaces containing $l_{1}$, Israel J. Math. 20 (1975), 375-384.

13. C. Stegall, The Radon-Nikodym property in conjugate Banach spaces. II, Trans. Amer. Math. Soc. 264 (1981), 507-519.

Department of MAThematics, University of British Columbia, VANCOUVER, BRITISH COLUMBIA, CANADA

Department of Mathematics, Université de Paris VII, Paris, France 